\title{
Three Dimensional Characterization of Xe Inclusions Ion-implanted in Al
}

\author{
M. Song ${ }^{*}$, H. Matsumoto ${ }^{* *}$, M. Shimojo ${ }^{* * * *}$, K. Mitsuishi ${ }^{*}$, and K. Furuya ${ }^{*}$ \\ *National Institute for Materials Science, Sakura 3-13, Tsukuba, Ibaraki, 3050003 Japan \\ **Yokohama Lab., Mitsubishi Chemical Group Science and Research Center, INC, 1000 Kamoshida-cho, \\ Aoba-ku, Yokohama, 2278502, Japan. \\ ${ }^{* * *}$ Saitama Institute of Technology, Fusaiji 1690, Fukaya, Saitama, 3690293 Japan
}

The precipitation of inert gas atoms in metals is interesting in views from both fundamental sciences and industrial applications, especially for nuclear materials. The microstructures of Xe-precipitates in Al have been observed with BF-TEM (bright field TE) and HRTEM (high-resolution TEM) [1]. The observed images usually contain only two dimensional (2-D) information on the structure and distribution of Xe precipitates in matrix. However, the 3-D nature of Xe-precipitates, the 2-D projection causes the overlapping of the interested structures such as precipitates and defects. Therefore, it is difficult to obtain detailed and correct information in 3-D way. TEM tomography is a powerful method to analyze the 3-D information of objects embedded in a matrix. We used a newly developed tomography specimen holder of a high voltage electron microscope (HVVEM). One of the features of this holder is that it utilizes an in-holder-rotation system, with which the rotation over $+/-90$ degrees can be easily made in the microscope. The detail of the holder system is described in this paper and others [2].

A rod-shaped Al specimen in diameter of about $100 \mathrm{~nm}$ was prepared with FIB (focused-ion-beam) and $\mathrm{Ar}^{+}$ion milling. The Xe-ion implantation was carried out with the ion-implanter-interfaced TEM, JEM-ARM1000 [3]. The TEM observations were carried out in BF mode and in rotation range from -90 to +90 degrees with an increment of 2.16 degrees. The TEM images recorded with CCD camera were reconstructed using the IMOD program developed by Mastronarde et al [4]. The experiments were performed at room temperature.

Fig. 1 shows typical TEM BF images taken at three different rotation angles. Round shaped contrasts shown by the arrows ( 1 and 2$)$ are Xe precipitates. In order to get better contrast of the precipitates, the micrographs were taken in overfocus condition. Fig. 2 shows a series of segments from the reconstructed tomogram of a single Xe nanoparticle. It is clear that the cross-sectional images by changing the depth in x-direction indicate the cubo-octahedral shape of the precipitate. Further experiments were done to analyze the precipitation behavior along the implantation depth. Figs. $3 \mathrm{a}$ and $3 \mathrm{~b}$ show schematic drawing of the direction-relationships of the TEM observation to the cross-sectional BF image of the specimen. The ion-implantations were performed in directions $\mathrm{A}$ and $\mathrm{B}$ as shown in Fig. $3 \mathrm{~b}$ to fluences of $4.6 \times 10^{15}$ and $1.5 \times 10^{15}$ ions $\mathrm{cm}^{-2}$, respectively. The nanosized Xe-precipitates, some of them are as small as about $2 \mathrm{~nm}$, and the distribution of the precipitates in 3 cross-section planes, are well identified. Note that almost no missing wedge exists. The distances between the precipitates and the sizes of the precipitates were measured directly from the segments. The image in Fig. 3c clearly indicates unusually large precipitates, which caused by the local diffusion of implanted Xe-atoms and was resulted from coarsening happening during the ion-implantation. Moreover, Fig. 3d implies another interesting behavior of Xe precipitates, which exist in the extended depth beyond the range of Xe ions (about $21 \mathrm{~nm}$ ).

\section{References}

[1] M. Song, K. Mitsuishi and K. Furuya, Mat. Sci. and Eng. A 304-306 (2001) p. 135.

[2] M. Song, H. Matsumoto, M. Shimojo, K. Furuya, and H. Sano, presented at EMC2008, Aachen, Sept. 1-5, 2008

[3] N. Ishikawa and K. Furuya, Ultramicroscopy 56 (1994) p. 211.

[4] D.N. Mastronarde, J. Struct. Biol. 120 (1997) p. 343; J.R. Kremer, D.N. Mastronarde and J.R. McIntosh, J. Struct. Biol. 116 (1996) p. 71; Also: http://bio3d.colorado.edu/imod/. 


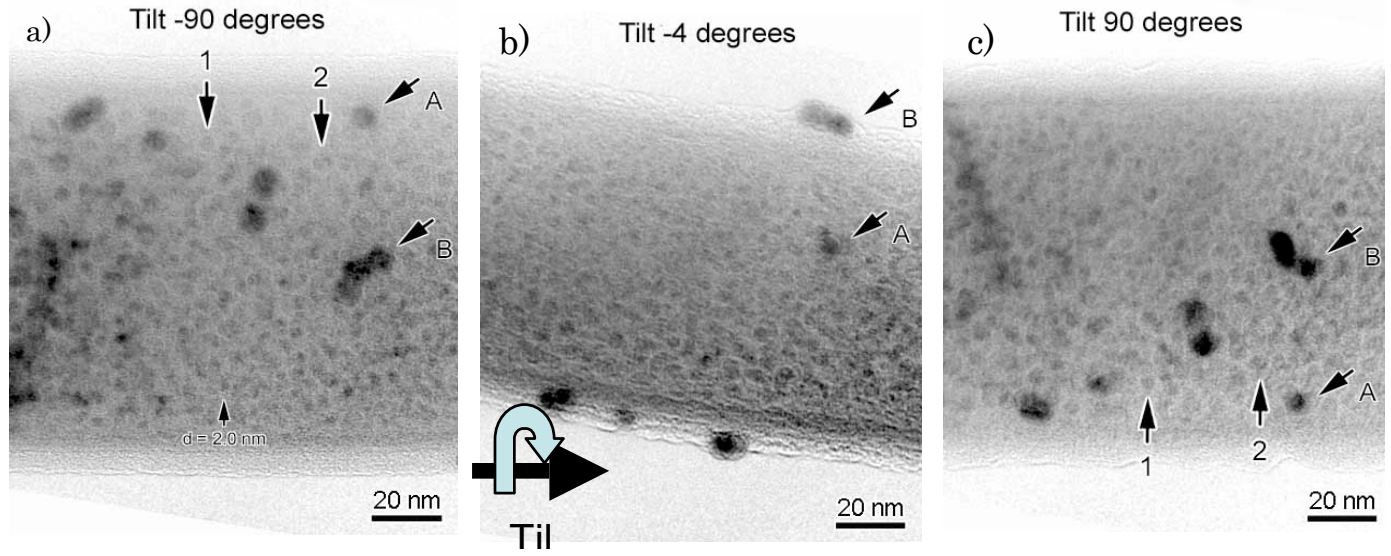

FIG. 1. Typical TEM BF micrographs of a Xe-implanted Al specimen at rotations of -90 (a), -4 (b) and +90 degrees (c). Arrows 1 and 2 show the same Xe particles, respectively. A and B show Au particle markers. -90 and +90 views are in mirror symmetry each other. Xe nanoparticles in high density are observed.
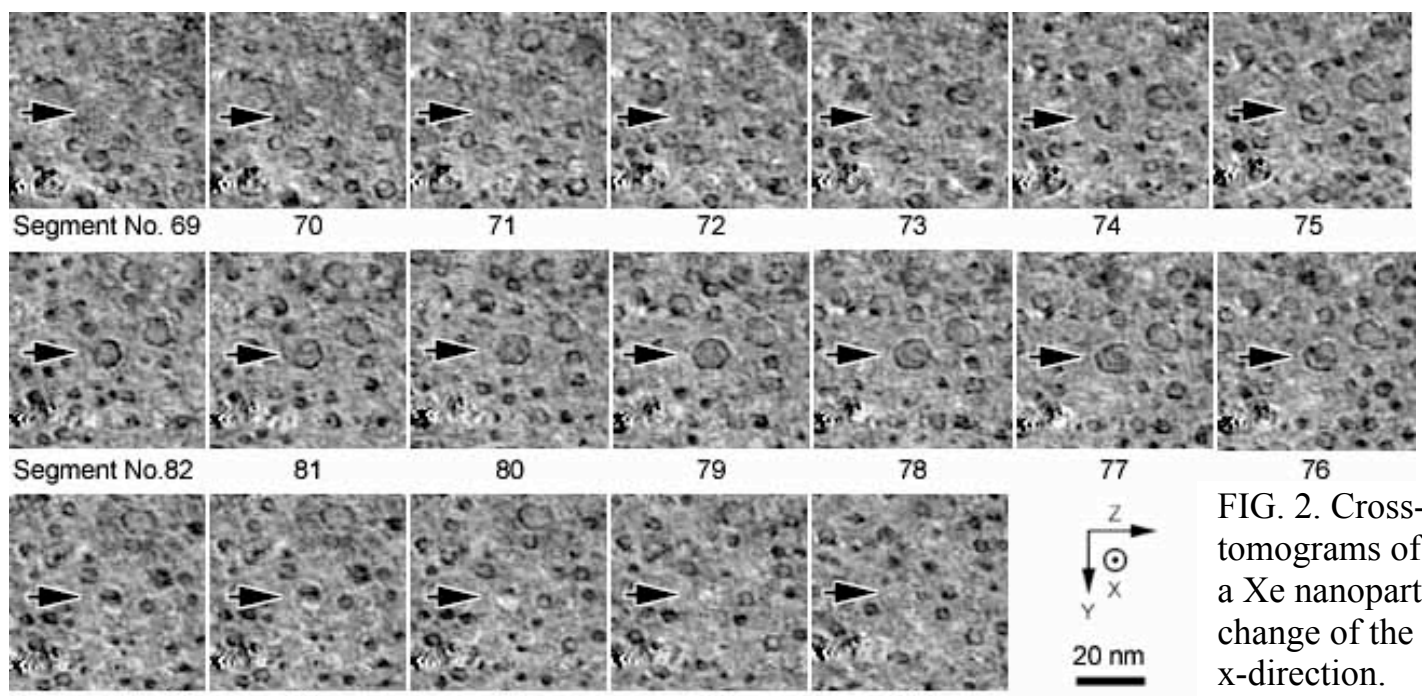

FIG. 2. Cross-sectional

tomograms of each segment of a Xe nanoparticle, showing the change of the shape in

Segment No. 83

84

a
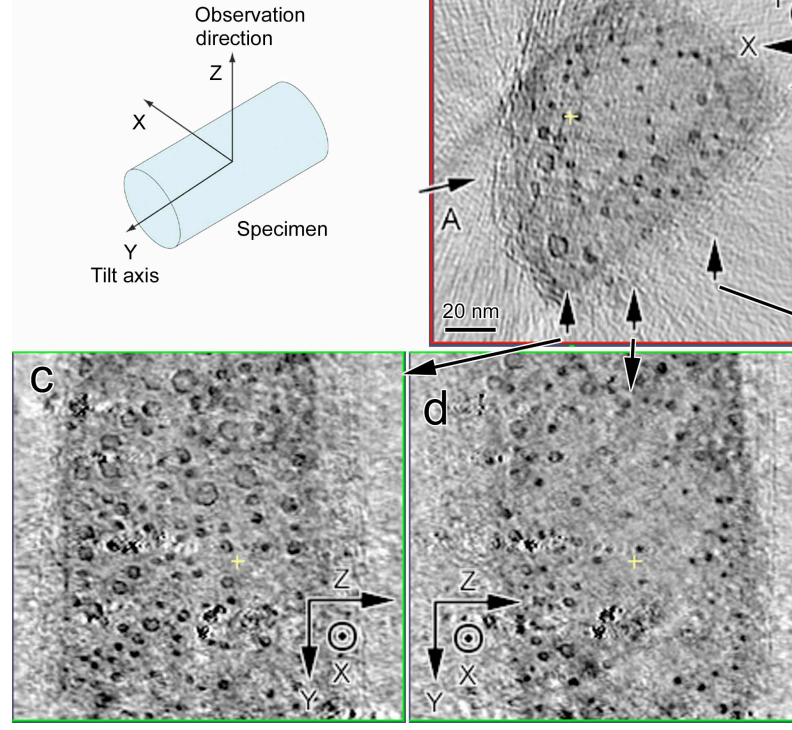

86
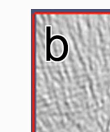

\begin{abstract}
85
\end{abstract}
87
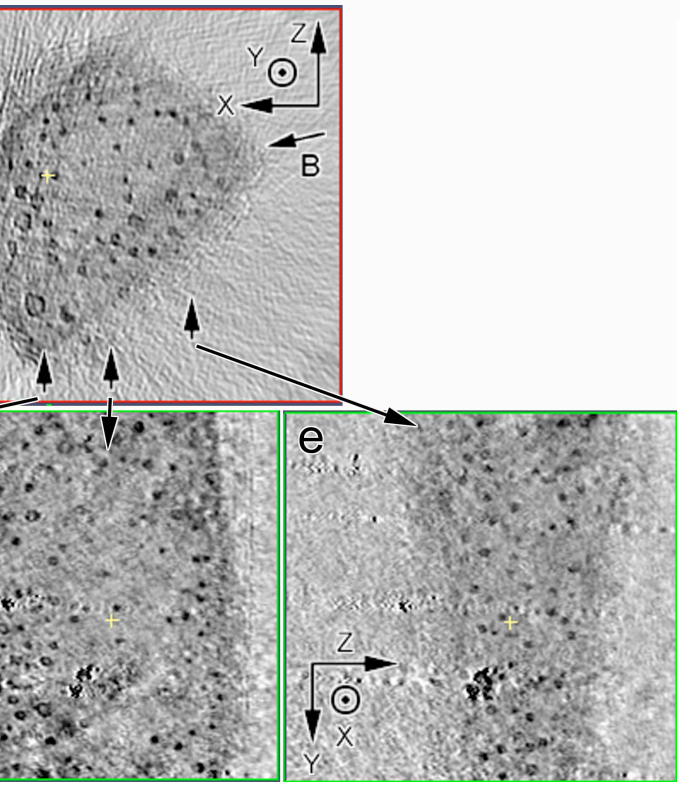

FIG. 3. The cross section views of the tomogram. (a) The configuration of sample. (b) The cross-sectional BF image of the sample. (c) Xe particles near the peak of ion range in the high dose side which is obviously larger than that in (e). The very large particles in (c) is resulted from the coalescence of usual size Xe particles. (d) Xe nanoparticles formed extended depth beyond the range of Xe ions. 